\title{
Cohérence d'une déviance : la mise en place de l'enseignement de l'anglais dans un DESS de gestion des industries du luxe et des métiers de l'art
}

\section{Nicole Terrien}

\section{(2) OpenEdition}

\section{Journals}

Édition électronique

URL : http://journals.openedition.org/asp/3912

DOI : 10.4000/asp.3912

ISSN : 2108-6354

Éditeur

Groupe d'étude et de recherche en anglais de spécialité

Édition imprimée

Date de publication : 1 décembre 1995

Pagination : 253-261

ISSN : 1246-8185

Référence électronique

Nicole Terrien, «Cohérence d'une déviance : la mise en place de l'enseignement de l'anglais dans un DESS de gestion des industries du luxe et des métiers de l'art », ASp [En ligne], 7-10 | 1995, mis en ligne le 30 octobre 2013, consulté le 20 avril 2019. URL : http://journals.openedition.org/asp/3912 ; DOI : 10.4000/asp.3912

Ce document a été généré automatiquement le 20 avril 2019.

Tous droits réservés 


\title{
Cohérence d'une déviance : la mise en place de l'enseignement de l'anglais dans un DESS de gestion des industries du luxe et des métiers de l'art
}

\author{
Nicole Terrien
}

Loxos, en grec signifie oblique, louche; d'où la notion de déviance. La définition même du mot luxe peut s'appliquer à celle du diplôme qui le représente dans le monde universitaire. Il s'agit d'un DESS de gestion faisant place aux habituelles disciplines enseignées dans ce contexte mais chaque fois adaptées à l'univers spécifique du luxe. Les études de cas portent uniquement sur des entreprises de ce secteur certes, mais il existe aussi des cours spécifiques sur le marketing des industries du luxe, le droit des marques, la gestion de la création, la gestion de l'innovation, l'histoire de l'art et l'histoire de la mode... La cohérence vient de la volonté appuyée de cibler le sujet sous ses aspects les plus divers tout en respectant les catégories généralement admises dans ce type de formation. La place privilégiée de l'anglais traduit cette adaptation à une culture très particulière.

2 Créé à la rentrée universitaire 1992 à l'université de Marne-la-Vallée, le diplôme est né sur la proposition de Madame Danielle Allérès, titulaire d'un doctorat d'État sur la gestion des entreprises de cosmétiques, directrice de la formation, pour répondre à une demande d'entreprises capables d'embaucher, malgré les restrictions économiques, des cadres directement opérationnels dans un domaine jusqu'alors négligé par les formateurs, dans le secteur public du moins. Une université nouvelle croyant aux diplômes à bac +5 et ayant besoin de se forger une image de marque novatrice semblait l'endroit idéal pour lancer une formation inédite. Sur place, quelques enseignants contactés par Mme Allérès ont accepté le défi et se sont ralliés à une équipe opérant déjà dans d'autres diplômes de gestion. Une campagne de presse fut donc lancée dans les mois précédant la rentrée, les candidatures affluèrent rapidement. Étant donné le caractère un peu rapide de la décision 
d'ouvrir le diplôme dès septembre 1992, les premières candidatures provinrent surtout des universités parisiennes mises au courant, Assas et Dauphine, et des écoles de commerce. Les deux années suivantes on a constaté une ouverture sur la province et des formations plus variées, même si la majorité des candidats ont une maîtrise de gestion, économie ou droit, ou un diplôme d'école de commerce, on voit quelques LEA, littéraires égarés, AES...

Une procédure de sélection fut mise en place pour limiter les effectifs à un nombre raisonnable pour une promotion pionnière : 25 étudiants (nombre conservé pour les deux promotions suivantes). Une audition, menée par Mme Allérès avec quelques enseignants et des représentants des entreprises, met à jour les motivations des candidats et leurs aptitudes à s'adapter au milieu du luxe.

Un oral d'anglais complète la sélection et sert de veto éventuel. Les candidats écoutent un très bref enregistrement de nouvelles, doivent en rendre compte, apporter un commentaire rapide, puis répondre à quelques questions sur leur formation initiale et leurs motivations, en anglais. Le but de ce test n'est pas de noter des connaissances mais d'évaluer des aptitudes à suivre un enseignement en anglais et à accepter une autre culture. Il est tenu compte de la très grande inégalité des formations suivies; les candidats en formation permanente sont jugés avec une grande mansuétude. Les tests ne dépassent pas dix à quinze minutes. En 1994 des enregistrements du même type que ceux destinés aux écoles de commerce ont remplacé les informations radiophoniques; les résultats ainsi obtenus sont comparables mais donnent plus l'impression aux candidats de subir un examen. À quelques notables exceptions près, les candidats se débrouillent en anglais, c'est-à-dire qu'ils comprennent le gros de l'information et qu'ils se font comprendre. Les lacunes essentielles concernent la précision tant de la compréhension que de l'expression. À bac + 5, il est temps d'acquérir le sens des nuances qui font basculer les négociations. Le but de l'enseignement sera donc de démontrer la nécessité de la précision et d'imposer cette nouvelle norme, de donner un cadre de références culturelles que chacun développera en fonction de ses activités et de ses goûts, d'effacer les jugements sommaires (si faciles dans des domaines sensibles comme la mode, la gastronomie, les cosmétiques...), de relativiser des valeurs que nos étudiants croient universelles. Une reprise systématique des points de grammaire n'est pas exclue d'une telle démarche. Le cours de langue garde son aspect technique, cependant il ne s'agit plus d'acquérir un nouvel outil, mais d'apprendre à s'en servir de façon efficace et donc intelligente. Il faut répondre à la double nécessité d'être spécifique et généraliste, technique et culturel, montrer qu'à partir d'un vocabulaire de base on peut élaborer un lexique spécifique en situation.

\section{Le statut de l'anglais}

5 Cinquante heures ont été allouées à l'anglais la première année, puis quarante-cinq heures pour l'anglais du luxe et des métiers de l'art, complétées par vingt heures pour l'anglais de la négociation et de la gestion (coefficient 2), à partir de la seconde année, ce qui a permis de faire appel à deux enseignants différents. Le coefficient 6 donné à la note d'anglais, qui comme les autres est éliminatoire à 8 et au-dessous, assure le respect de la matière. Seul le marketing des industries du luxe a un coefficient supérieur, le total des coefficients étant de 40. L'anglais dit du luxe donne seul lieu à deux examens, l'un en décembre, l'autre en février. 
6 Pour souligner l'importance de l'anglais, il a été décidé que les cours commenceraient dès la seconde semaine de septembre sous forme de cours intensifs, à raison de trois heures par jour, quatre jours par semaine. La première année ce stage a duré trois semaines, ce qui a permis au groupe de se souder très rapidement autour d'une matière perçue à la fois comme ludique et comme essentielle. Cependant la fatigue certaine qui résulte d'une telle pratique et l'épuisement trop rapide du quota d'heures/langue ont conduit à une révision vers deux semaines intensives suivies d'un cours hebdomadaire de 1 heure 30 jusqu'à début février. Cette solution permet de mieux étaler les apprentissages, mais elle banalise aussi l'anglais tout en remettant en cause le découpage initial choisi entre les trois grands thèmes : la mode, les cosmétiques, l'art, une semaine étant consacrée à chaque domaine représentant les centres d'intérêt principaux des étudiants et les rubriques traitées dans les autres matières. Dans le schéma des deux semaines intensives ont été conservés les cours sur la mode et l'art, de sorte à souligner la double appellation du diplôme. Le cours sur les cosmétiques se trouve éclaté ; sans disparaître, il perd toutefois de sa force.

\section{La mode, d'un lexique à un métier}

7 Il faut démontrer de façon immédiate que ce cours est totalement intégré au cursus pour que les étudiants en relative difficulté ne se découragent pas a priori et que les autres ne s'endorment pas sur un passé faussement glorieux. Puisque la fascination pour l'univers de la mode apparait comme essentielle dans les entretiens avec les candidats, on les plonge directement dans ce rêve en visionnant des enregistrements de défilés de mode. Deux exercices s'ouvrent alors: un exercice de compréhension pour restituer le commentaire du journaliste, un exercice d'expression pour forger une description des modèles observés.

\section{Compréhension}

8 Les différences d'accents, britanniques ou américains avec de fortes caractéristiques régionales, posent problème et exigent une concentration plus grande que pour des intonations universitaires. Les interviews de créateurs, de mannequins ou d'autres journalistes spécialisés convainquent du caractère réaliste de l'exercice. Il est impossible de rejeter les difficultés en se disant qu'elles appartiennent à une catégorie socioprofessionnelle éloignée. L'association d'un vocabulaire spécifique et d'expressions courantes, voire figées, souligne le lien entre l'anglais courant et le nouveau lexique à acquérir. Les étudiants notent l'utilisation exagérée des formules hyperboliques et des adjectifs sur le goût et se rendent compte du caractère ridicule de jugements a priori sans justification, ce qui rendra plus faciles les futures critiques de l'enseignant sur leurs propres tendances à la facilité.

9 Les prestations de personnalités françaises dans un anglais approximatif, énoncé avec un accent parisien certain, participent également à une prise de conscience importante en début de cursus. 


\section{Au niveau de l'expression}

10 Une fois compris, ces commentaires donnent lieu à comparaisons afin de souligner les divergences d'approche en fonction des cultures, de mettre à jour les préjugés auxquels nos étudiants devront faire face une fois en situation. Parallèlement les productions des divers pays reflètent des mentalités différentes et une approche divergente de l'industrie de la mode elle-même. On peut donc déjà suggérer un premier lien entre les cultures nationales et les cultures d'entreprises, d'autant que les commentaires abondent sur l'image que l'on a de soi et de l'image que l'on cherche à projeter.

11 Les étudiants doivent proposer, par oral, leur propre commentaire du défilé. Il est évident que la précision du lexique sur le vêtement fait défaut, mais surtout la pauvreté des adjectifs sur les couleurs, les matières, les impressions souligne la tendance à se contenter toujours d'un vocabulaire approximatif. La mise en commun des ressources de chacun montre qu'il ne s'agit pourtant pas d'un exercice impossible, mais qu'il faudrait réactiver un vocabulaire passif accumulé au cours des études passées. Cet exercice développe l'entraide et la modestie des meilleurs qui ne sont pas parfaits. Les étudiants de formation permanente qui possèdent souvent un vocabulaire plus traditionnel participent activement. Cet exercice de mise en forme assure le lien avec la seconde partie du cours qui opère sur support écrit.

\section{Support écrit}

Il s'agit, le plus souvent, d'un compte-rendu dans la presse des défilés que l'on a vus sur écran. Ces articles font nécessairement appel à une plus grande précision de vocabulaire pour compenser l'absence de l'image. Le recul pris entre le moment du défilé et le moment de l'écriture autorise également une ébauche d'analyse des tendances et une comparaison entre les diverses scènes de la mode. Les étudiants retrouvent l'exercice qui leur a été demandé et pensent à réutiliser ce modèle lors du cours suivant.

13 Ces textes sont complétés par une série d'articles de fond sur les industries de la mode de façon à élargir vers le marketing, la vente, l'étude des circuits de distribution et à faire le lien avec des données économiques plus générales. The Economist, Fortune, parfois Time sont les sources les plus fréquemment utilisées. Les étudiants lisent le texte chez eux et doivent proposer un résumé. Cet exercice est loin d'être maîtrisé et il révèle de sérieuses différences entre les formations d'origine : les scientifiques, les étudiants de LEA et des écoles de commerce discernent assez facilement les informations essentielles, les autres, économistes ou gestionnaires formés à l'université, se perdent dans les détails. Il a donc été décidé de répéter cet exercice à chaque cours, ce qui se révèle efficace. Un commentaire fait suite, qui mène vers un élargissement. Les étudiants sont satisfaits de pouvoir réutiliser en anglais ce qu'ils ont appris dans d'autres matières.

14 Quelques articles chers à la presse anglo-saxonne sur les role-models complètent cette approche et accentuent les différences culturelles en décrivant les comportements de grands patrons, souvent américains. Fortune est une source précieuse; ces articles plaisent aux étudiants de formation permanente plus sensibles aux problèmes des relations humaines, les autres sourient parfois. Ces articles étant plus longs donnent lieu à des résumés écrits. 
La lecture de l'article sur la mode de l'Encyclopedia Britannica, en début ou en fin de cycle, assure un lien avec des idées plus abstraites, des notions historiques et philosophiques (sans prétentions exagérées).

\section{L'art, un marché sur le métier}

L'utilisation de la vidéo sur la mode continue lors de cette semaine, mais en guise de détente dans la dernière partie du cours. Une certaine habitude s'est créée qui rend les exercices moins astreignants.

Le cours d'histoire de l'art n'intervenant que plus tard dans l'année, on ne peut faire appel qu'à une culture générale très variable d'un étudiant à l'autre. L'art est abordé sous forme de dossiers mettant en relation l'aspect institutionnel et l'aspect commercial. Il s'agit de présenter les grandes structures d'encadrement propres aux États-Unis et à la Grande-Bretagne, à travers une comparaison avec le système français, système qu'il faut définir à grands traits, car les étudiants le connaissent mal en début d'année.

On voit que la centralisation du système français ne correspond pas du tout à la volonté d'autonomie en place aux États-Unis et en cours de réalisation en Grande-Bretagne. L'accent est mis sur la nécessité de se plier à des règles de rentabilité relative et sur les difficultés à faire coïncider le culturel et l'économique. Le vocabulaire de la gestion et les notions qu'il exprime sont mis en pratique. Les étudiants sont ainsi obligés d'abandonner un discours lénifiant sur les valeurs culturelles pour passer à un véritable examen des mentalités et des institutions.

Sans avoir la prétention d'apporter des réponses à tous les problèmes, cet exercice de comparaison éveille du moins l'esprit critique. Par exemple la gestion des musées dans le système américain présente le mérite d'éviter le gaspillage des deniers publics, mais introduit le danger de sacrifier à la facilité. De même, la comparaison entre les productions théâtrales aux États-Unis et en Grande-Bretagne marque-t-elle le conflit entre la créativité et la culture de masse. Les étudiants sont bien amenés à s'interroger sur des notions qu'ils ont naturellement tendance à considérer comme acquises.

Le discours sur les institutions présentant l'art devient un moyen d'envisager les différentes réformes que pourrait, à plus ou moins long terme, impliquer l'Union européenne et qu'entraîne la concurrence internationale. Un domaine sensible évident en celui du marché de l'art, à travers les ventes aux enchères, les réformes subies par Christie's et Sotheby's au cours des dernières années, la remise en cause d'une certaine éthique autour des problèmes de contrefaçon ou de vols. Cette étude souligne le lien entre le monde de l'art et celui de la gestion des entreprises. Un minimum de connaissances est exigé sur les institutions, les lois et les mentalités. La réflexion sur les politiques culturelles croise les problèmes rencontrés dans tous les secteurs du luxe au sujet de la protection de la propriété intellectuelle ou de la créativité. Quelques exemples de mécénat soulignent les relations étroites entre les deux univers.

21 Le lien entre les deux grandes sections du cours s'opère non seulement au niveau culturel abstrait mais bien au niveau de l'application technique. Le cours de langue dispose les étudiants à recevoir un enseignement très précis dans les matières spécifiques à ce DESS, enseignement qui, à son tour, enrichira le discours tenu en cours d'anglais plus tard dans l'année. 


\section{Les cosmétiques : une explosante fixe} entre les thèmes, mais aussi entre les aspects de la langue étudiés. Le but des autres cours est de consolider ce tissu tout en répondant aux goûts des diverses promotions. Les textes d'illustration insisteront donc sur l'aspect économique et commercial des thèmes abordés en fonction de la demande : hôtellerie de luxe (enquête sur le bien-être des clientes, point sur le regroupement des chaînes hôtelières, choix des prestations et des localisations, comparaisons nationales), accessoires (influence de la mode et des conditions économiques), décoration (choix culturels, rapport à l'histoire), architecture (relation avec le mode de vie, avec l'image de marque), arts de la table (traditions et innovations)... Plusieurs articles sur les systèmes de distribution et leur évolution tendent à souligner la spécificité de la distribution de luxe, qui représentera le principal débouché de nos étudiants.

\section{Évaluation}

Les impératifs du diplôme imposent un contrôle final écrit. Un premier examen a lieu en décembre, portant sur les acquis de la première semaine dans le domaine de la mode, complétés par quelques textes sur la distribution. Les étudiants doivent résumer un article de presse (à tendance économique) et répondre à deux ou trois questions les menant à une analyse de la situation actuelle et des tendances du marché. On peut souvent regretter la lenteur du travail et un manque de confiance en soi dès qu'il s'agit d'un examen, qui contraste avec la spontanéité des réponses en cours. Les étudiants se 
réfugient dans un discours peu précis plutôt que de prendre le risque de soutenir un point de vue personnel ; en revanche, il y a un effort certain de réutilisation du lexique.

Le deuxième examen a lieu en février et porte sur le domaine de l'art. L'insistance sur le marché permet de réintroduire les notions développées à propos d'autres thèmes, les étudiants sont invités à établir des comparaisons entre art et luxe. Ce deuxième examen leur semble généralement plus facile, certainement parce qu'ils ont pris du recul et ont assimilé de nombreuses notions dans les autres cours.

Les résultats d'ensemble sont satisfaisants; le public est motivé et progresse donc. Cependant, ces quelques mois de cours ne suffisent pas à effacer les différences initiales : on peut espérer qu'ils auront confirmé un goût pour une culture multiple et un sens de l'effort d'adaptation.

2 Les échos reçus des anciens étudiants sont encourageants. Presque tous se servent de l'anglais et déclarent avoir appris à mobiliser leurs connaissances. La pratique intensive du début d'année leur semble avoir été essentielle dans le passage vers l'utilisation courante de la langue hors cadres universitaires.

La plupart d'entre eux ont trouvé un emploi, malheureusement souvent sous la forme de CDD. Quelques réussites notoires nous paraissent encourageantes: une création d'entreprise s'occupant de l'organisation de manifestations musicales, une étudiante est devenue, à l'issue de son stage, Directrice de l'École de Broderie Lesage (broderie haute couture), une autre dirige le service marketing de MacDouglas France, une autre est chargée de la réorganisation du marketing des Boutiques des Aéroports de Paris... Ces emplois ont été trouvés grâce aux stages et non en fonction des relations personnelles. Il faut noter que les deux premiers majors sont une ancienne LEA et un ancien AES. Un échec chaque année a marqué les deux premières promotions qui sont fières de porter un nom prestigieux : Nina Ricci, Garouste et Bonetti, la troisième s'appellera Christian Dior.

À noter pour l'université un prestige certain puisque des personnalités comme Paco Rabane acceptent de se déplacer pour faire des conférences et surtout que la formation est celle qui rapporte à Marne-la-Vallée la plus grande taxe d'apprentissage.

\section{La recherche}

31 Parallèlement au DESS a été créée une jeune équipe de gestion. Pour des raisons obscures, l'administration n'a pas souhaité sa reconduction. L'équipe s'est alors reconstituée sous loi de 1901 chez Jean Patou. Elle avait en 1993-1994 donné lieu à une série de communications dont une sur la littérature en langue anglaise. Les membres de l'équipe s'étaient déclarés intéressés par un compte rendu qui pourrait servir de base de données sur les représentations des nouvelles formes du luxe en littérature de langue anglaise, marquant ainsi la reconnaissance de l'anglais comme discipline de recherche et confirmant l'objectif du cours qui est de démontrer la nécessité d'analyser les mentalités et les représentations de soi afin de mieux cerner le public auquel une industrie destine sa production. L'anglais est bien l'outil dont on se sert pour pénétrer le marché angloaméricain qui ne répond pas aux mêmes sollicitations que le marché français. Il y a totale reconnaissance des capacités d'analyse de chacun. Cette année, ma collègue angliciste développera l'exemple de la production de bijoux dans la tribu Navajo. On retrouve le croisement avec l'enseignement et la recherche en civilisation. 
Plusieurs étudiants ont déjà manifesté leur désir de prolonger leur formation sur le luxe par un doctorat spécifique. Si l'administration acceptait les inscriptions, la gestion des industries du luxe et des métiers de l'art deviendrait à part entière une matière universitaire et contribuerait mieux que tout discours à l'ouverture de l'université sur le monde de l'entreprise.

\section{RÉSUMÉS}

À travers la description du cours mis en place pour ce diplôme unique, il s'agit de repérer le rôle que peut jouer l'enseignement de l'anglais de spécialité au croisement de la langue technique et $\mathrm{du}$ véhicule culturel. Le lexique technique sert de support à une approche globale qui utilise systématiquement les particularités culturelles. Les étudiants sont invités à préciser leur compréhension et à affiner leur expression dans le but de convaincre un interlocuteur étranger. Cet exemple illustre la nécessité de ne jamais séparer texte et contexte dans le souci de rendre actif l'outil qu'est l'anglais de spécialité.

Through the description of the course established for a degree that is still unique in the French university system, the author of this paper has underlined that the English class teaches a technical language while constantly referring to cultural specificities. The acquisition of technical vocabulary is part of a global approach and the students are invited to improve their understanding in order to express themselves more accurately whenever they have to convince a foreign interlocutor. This case shows how important it is to insist on the cultural background if we want the teaching of English to reach its very specific purpose.

\section{INDEX}

Mots-clés : art, culture, lexique spécialisé, luxe

Keywords : luxury, technical lexicon

\section{AUTEUR}

\section{NICOLE TERRIEN}

Ancienne élève de l'École normale supérieure de Fontenay-aux-Roses, agrégée d'anglais, docteur de $3^{\mathrm{e}}$ cycle, Nicole Terrien enseigne depuis 1989 comme maître de conférences à l'université de Marne-la-Vallée où elle a créé le département de LEA, puis de LCE. Auteur de plusieurs articles sur la littérature du $20^{\mathrm{e}}$ siècle, elle a proposé l'utilisation de références littéraires comme base de données pour une réflexion sur l'évolution de la notion de luxe. Sa communication (1994) « Nouveaux riches, nouveaux luxes : quelques exemples dans la littérature de langue anglaise des $19^{\mathrm{e}}$ et $20^{\mathrm{e}}$ siècles » paraîtra prochainement. Nicole.Terrien@univ-rouen.fr 